\title{
Anesthesiomics: Could a New Name Be Coined for Anesthesia?
}

\author{
Ali Dabbagh (iD) ${ }^{1}{ }^{*}$ \\ ${ }^{1}$ Anesthesiology Department, Anesthesiology Research Center, School of Medicine, Shahid Beheshti University of Medical Sciences, Tehran, Iran \\ "Corresponding author: Anesthesiology Research Center, Shahid Beheshti University of Medical Sciences, Tehran, Iran. Email: alidabbagh@yahoo.com
}

Received 2020 January 12; Accepted 2020 January 16.

Keywords: Anesthesiology, Personalized Medicine, Anesthesiomics

\section{Dear Editor,}

In recent years, the revolutionary trend in personalized medicine has affected almost every aspect of the medical science. The practice of personalized medicine is based primarily on translational research results (1, 2 ). In this path, novel aspects of medicine, including but not limited to metabolomics, proteomics, genomics, epigenomics, transcriptomics, interactomics and other "OMICS", are gradually replacing older medical approaches and philosophies of medicine. Nowadays, novel terminology is coined not only for increasing the medical nomenclature, but also to be representative of current practical aspects of medicine (3-5).

The practice of anesthesiology and perioperative medicine has been targeted by "OMICS sciences" for the last couple of years (6). A detailed list of drugs is used in anesthesiology and perioperative medicine. In addition, many aspects of cellular and molecular function and body structure as well as many organ systems are affected by the process of anesthesia and perioperative management. As a result, the role of anesthesiologists and perioperative medicine specialists is one of the most related clinical applications of personalized medicine. To overstress the latter, note the increasing trend for creation and production of novel drugs based on the OMICS approach, the overwhelming use of electronic patient files and electronic anesthesia records and big data management systems for patients undergoing anesthesia (7).

In fact, with the tremendous speed of scientific improvements, and also, with the inevitable role of information technology (health IT) on transformation speed of medicine, the above change is an obligatory "paradigm shift" in medical practice. The speed of improvement has led to a change from "high cost" to "low cost" technology available not only for developed countries, but also for low and middle income countries.

According to futurology lessons, it is better to be proac- tive against changes. As once in 1840's, using the word "anesthesia" was uncommon and now it is a well-known branch of medicine and a routine clinical practice, possibly, we will not have to wait for more than a couple of years before the words "anesthesiomics", "personalized anesthesia", "personalized perioperative medicine" and "P4 perioperative medicine" become prevalent clinical words. Thus, we have to be highly prepared for this paradigm shift worldwide to better serve our patients.

\section{Footnotes}

Authors' Contribution: All parts of the letter are written only by Ali Dabbagh.

\section{Conflict of Interests: None.}

Funding/Support: None.

\section{References}

1. Kharasch ED. The challenges of translation. Anesthesiology. 2018;128(4):693-6. doi: 10.1097|ALN.0000000000002122. [PubMed: 29533965].

2. Davidson A. Translational research: What does it mean? Anesthesiology. 2011;115(5):909-11. doi: 10.1097|ALN.0b013e3182337a5e. [PubMed: 21934485].

3. Iravani M, Lee LK, Cannesson M. Standardized care versus precision medicine in the perioperative setting: Can point-of-care testing help bridge the gap? Anesth Analg. 2017;124(4):1347-53. doi: 10.1213/ANE.0000000000001663. [PubMed: 28027087].

4. Whitcomb DC. What is personalized medicine and what should it replace? Nat Rev Gastroenterol Hepatol. 2012;9(7):418-24. doi: 10.1038/nrgastro.2012.100. [PubMed: 22614753]. [PubMed Central: PMC3684057].

5. Dabbagh A, Elyassi H. Cellular and molecular anesthesia: From bench to bedside. J Cell Mol Anesth. 2016;1(1):1-2.

6. Olivier M, Asmis R, Hawkins GA, Howard TD, Cox LA. The need for multi-omics biomarker signatures in precision medicine. Int J Mol Sci. 2019;20(19). doi: 10.3390/ijms20194781. [PubMed: 31561483]. [PubMed Central: PMC6801754].

7. Simpao AF, Ahumada LM, Rehman MA. Big data and visual analytics in anaesthesia and health care. Br J Anaesth. 2015;115(3):350-6. doi: 10.1093/bja/aeu552. [PubMed: 25627395]. 